\title{
Predicting Amyloid Burden to Accelerate Recruitment of Secondary Prevention Clinical Trials
}

\author{
O. Langford ${ }^{1}$, R. Raman ${ }^{1}$, R.A. Sperling ${ }^{2}$, J. Cummings ${ }^{3}$, C.-K. Sun ${ }^{1}$, G. Jimenez-Maggiora ${ }^{1}$, P.S. Aisen ${ }^{1}$, \\ M.C. Donohue ${ }^{1}$ and the TRC-PAD Investigators*
}

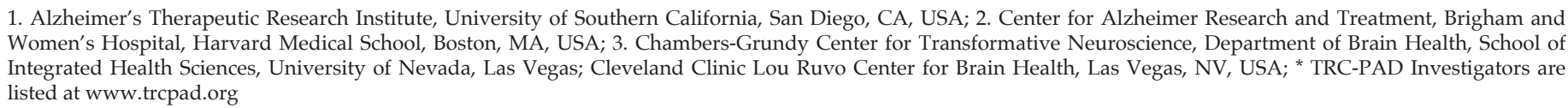

Corresponding Author: M.C. Donohue, Alzheimer's Therapeutic Research Institute, University of Southern California, San Diego, CA, USA, mdonohue@usc.edu

Published online August 11, 2020, http://dx.doi.org/10.14283/jpad.2020.44

\begin{abstract}
BACKGROUND: Screening to identify individuals with elevated brain amyloid $(\mathrm{A} \beta+)$ for clinical trials in Preclinical Alzheimer's Disease (PAD), such as the Anti-Amyloid Treatment in Asymptomatic Alzheimer's disease (A4) trial, is slow and costly. The Trial-Ready Cohort in Preclinical/ Prodromal Alzheimer's Disease (TRC-PAD) aims to accelerate and reduce costs of $\mathrm{AD}$ trial recruitment by maintaining a web-based registry of potential trial participants, and using predictive algorithms to assess their likelihood of suitability for
\end{abstract} PAD trials.

OBJECTIVES: Here we describe how algorithms used to predict amyloid burden within TRC-PAD project were derived using screening data from the A4 trial.

DESIGN: We apply machine learning techniques to predict amyloid positivity. Demographic variables, APOE genotype, and measures of cognition and function are considered as predictors. Model data were derived from the A4 trial.

SETTING: TRC-PAD data are collected from web-based and in-person assessments and are used to predict the risk of elevated amyloid and assess eligibility for AD trials.

PARTICIPANTS: Pre-randomization, cross-sectional data from the ongoing A4 trial are used to develop statistical models.

MEASUREMENTS: Models use a range of cognitive tests and subjective memory assessments, along with demographic variables. Amyloid positivity in A4 was confirmed using positron emission tomography (PET).

RESULTS: The A4 trial screened $\mathrm{N}=4,486$ participants, of which $\mathrm{N}=1323(29 \%)$ were classified as $\mathrm{A} \beta+(\mathrm{SUVR} \geq 1.15)$. The Area under the Receiver Operating Characteristic curves for these models ranged from 0.60 (95\% CI 0.56 to 0.64$)$ for a web-based battery without APOE to 0.74 (95\% CI 0.70 to 0.78 ) for an in-person battery. The number needed to screen to identify an $\mathrm{A} \beta+$ individual is reduced from 3.39 in $\mathrm{A} 4$ to 2.62 in the remote setting without $\mathrm{APOE}$, and 1.61 in the remote setting with APOE.

CONCLUSIONS: Predictive algorithms in a web-based registry can improve the efficiency of screening in future secondary prevention trials. APOE status contributes most to predictive accuracy with cross-sectional data. Blood-based assays of amyloid will likely improve the prediction of amyloid PET positivity.

Key words: Trial-ready cohort, Alzheimer's disease, machine learning.

\section{Background}

creening cognitively normal older individuals for the presence of elevated cerebral amyloidbeta protein ("A $\beta+$ ") and inclusion in secondary prevention trials for Alzheimer's disease (AD) is invasive, expensive and slow. The current gold standards to measure Amyloid- $\beta$ in the brain require either positron emission tomography (PET) or cerebrospinal fluid (CSF) assay. For example, the Anti-Amyloid Treatment in Asymptomatic Alzheimer's disease (A4) trial conducted amyloid PET on 4,486 individuals in order to identify $1,323 \mathrm{~A} \beta+$ individuals for an amyloid PET screen fail rate of $71 \%$ (1). The Number Needed to Screen (NNS) to identify each $\mathrm{A} \beta+$ individual was 3.39 individuals.

Trial-Ready Cohort in Preclinical/Prodromal Alzheimer's Disease (TRC-PAD) is a research program that was initiated to find solutions to these challenges in trial recruitment and site management, as described in Aisen, et al. Submitted (2). There are three elements that make up the TRC-PAD platform; Alzheimer's Prevention Trial (APT) webstudy (aptwebstudy.org), Site Referral System (SRS) and the Trial Ready Cohort (TRC). The APT webstudy invites participants to enroll into the study. At the time of enrollment, participants are asked for demographic, medical and lifestyle information. They are asked to complete longitudinal web-based cognitive testing and symptom questionnaires. With these data, we aim to estimate the likelihood that an individual is $\mathrm{A} \beta+$ before they are invited to participate in a secondary prevention trial. The SRS helps facilitate the participants deemed to be most likely $\mathrm{A} \beta+$ from APT to go for in-clinic assessments where they proceed with the TRC screening. During the TRC screening phase participants are administered additional testing, including Preclinical Alzheimer's Cognitive Composite (PACC) (3) and genotyping, before assessing their eligibility for an amyloid test.

In this paper, we describe how the prediction models and algorithms used in TRC-PAD were derived from A4 
Table 1. Predictors Considered

\begin{tabular}{|c|c|c|c|c|}
\hline Abbreviation & Variable & Description & Remote & In Clinic \\
\hline Age & Age & Number of years & $\sqrt{ }$ & $\sqrt{ }$ \\
\hline Edu & Education & Number of years & $\checkmark$ & $\checkmark$ \\
\hline Sex & Sex & Male or Female & $\checkmark$ & $\checkmark$ \\
\hline OCL & Cogstate One Card Learning & Accuracy & $\checkmark$ & \\
\hline OBR & Cogstate One Back Reaction & Reaction time & $\checkmark$ & \\
\hline DER & Cogstate Detection Reaction & Reaction time & $\checkmark$ & \\
\hline IDR & Cogstate Identification Reaction & Reaction time & $\checkmark$ & \\
\hline $\mathrm{FH}$ & Family History & Family history a parent or sibling with $\mathrm{AD}$ & $\checkmark$ & \\
\hline FH P & Family History - Parent & Family history a parent with $\mathrm{AD}$ & & $\checkmark$ \\
\hline FH S & Family History - Sibling & Family history a sibling with AD & & $\checkmark$ \\
\hline CFI Pt & Cognitive Function Instrument - Participant & & $\sqrt{ }$ & $\checkmark$ \\
\hline CFI SP & Cognitive Function Instrument - Study Partner & & & $\checkmark$ \\
\hline ADL Pt & Activities of Daily Living - Participant & & & $\sqrt{ }$ \\
\hline ADL SP & Activities of Daily Living - Study Partner & & & $\sqrt{ }$ \\
\hline PACC & Preclinical Alzheimer Cognitive Composite & & & $\sqrt{ }$ \\
\hline APOE4 & $\mathrm{APOE} \varepsilon 4$ & & $(X / \sqrt{ })$ & $(X / \sqrt{ })$ \\
\hline
\end{tabular}

We considered predictive algorithms which could be applied to data captured either remotely via a web-based registry, or in the clinic (though all data in A4 was collected in clinic), as indicated in the table. In all we considered 6 models: (1) remote battery with APOE, (2) remote battery without APOE, (3) in clinic battery with APOE, (4) in clinic battery without APOE, (5) in clinic battery with individual PACC component scores and APOE, and (6) in clinic battery with individual PACC component scores without APOE.

screening data. We anticipate blood-based biomarkers will greatly improve predictions of amyloid positivity, and this is a focus of future work and an aim of TRCPAD. Predictors in the current analysis are limited to demographics, cognitive and functional assessments, and APOE genotype.

\section{Methods}

\section{Population and Study Design}

The study design and screening data for A4 have been previously described $(7,8)$ and Institutional Review Boards have approved both A4 and TRC-PAD studies. The A4 screening dataset contains $\mathrm{N}=4,486$ participants, of which $1323(29 \%)$ were classified as A $\beta+$. Amyloid PET imaging was conducted with florbetapir F18 and summarized by mean cortical standardized uptake value ratio (SUVR) relative to the whole cerebellum. Participants were considered eligible to continue screening for A4 based on an algorithm combining both quantitative SUVR $(\geq 1.15)$ and qualitative visual read performed at a central laboratory. A SUVR between 1.10 and 1.15 was considered to be elevated amyloid only if the visual read was considered positive by a two-reader consensus determination. Participants who were considered $A \beta+$ were slightly older; with mean/ standard deviation (SD) age of $72.10 / 4.89$ in the $A \beta+$ group and $70.95 / 4.53$ in the $A \beta$ - group. However, there were no observed differences in sex and education. $A \beta+$ participants were more likely to have a family history of dementia and at least one APOE 44 allele. In addition, $\mathrm{A} \beta+$ participants performed worse on the screening Preclinical Alzheimer Cognitive Composite (PACC) results and had higher scores on the Cognitive Function Index.

\section{Variables}

Table 1 describes the collections of predictors that we considered to train different predictive algorithms. All screening data for the A4 Study were collected during supervised clinic visits. However some components of the A4 screening battery are being captured remotely in the APT webstudy, including demographic, Cogstate brief battery (9), family history (sibling or parent with Alzheimer's), and Cognitive Function Instrument (10) (CFI) variables indicated in Table 1. We consider predictive algorithms using these "remote" variables only, as well as a more thorough battery that would require a supervised clinic visit with an administration of the PACC3. In all, we considered 6 models: (1) remote battery without APOE, (2) remote battery with APOE, (3) in clinic battery without APOE, (4) in clinic battery with APOE, (5) in clinic battery with individual PACC component scores without APOE, and (6) in clinic battery with individual PACC component scores with APOE. The PACC component scores include the MiniMental State Exam (MMSE) (11), Wechsler Memory ScaleRevised Logical Memory, Digit Symbol Substitution 
Figure 1. Contribution of 5 best predictors in each model
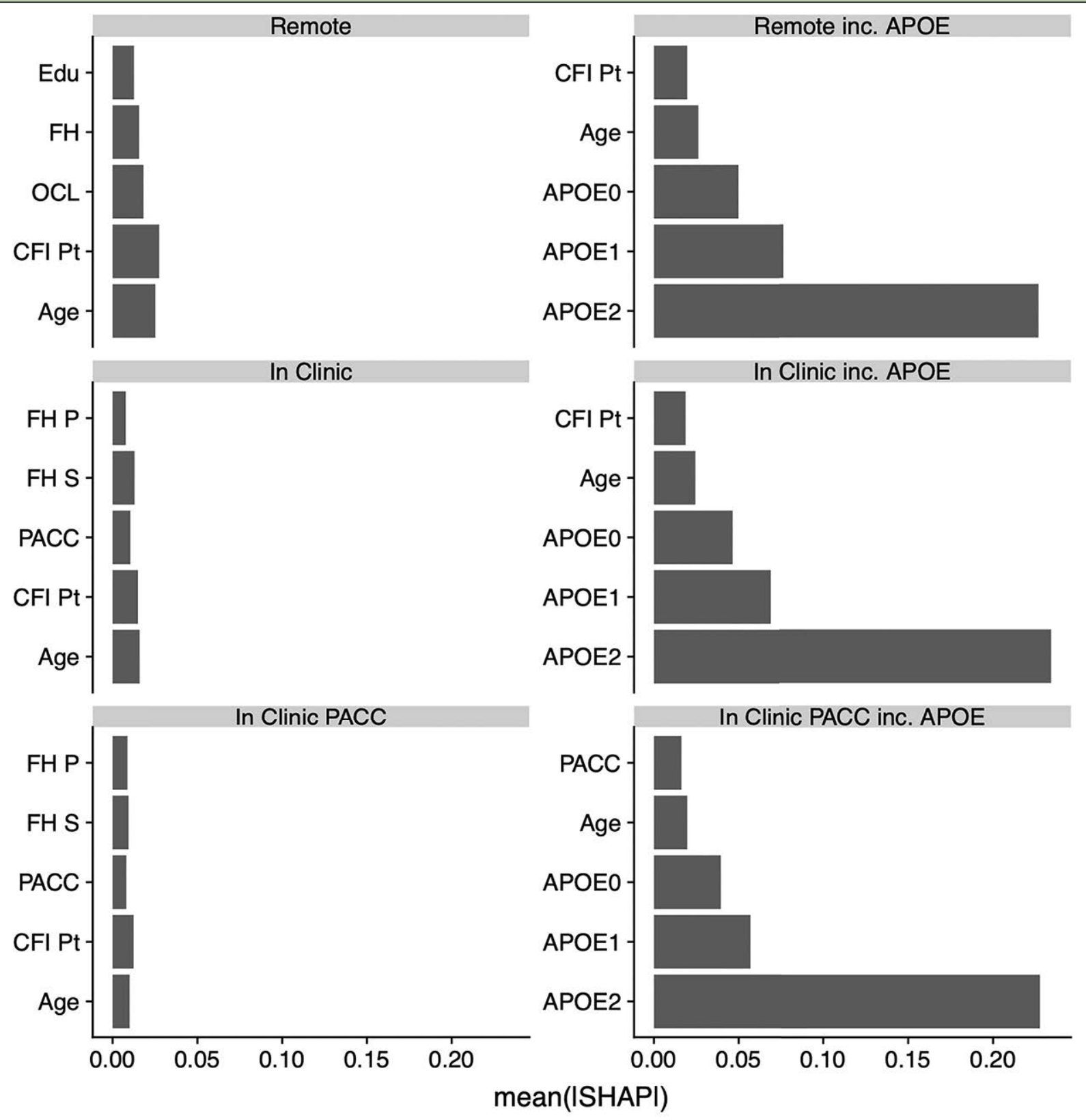

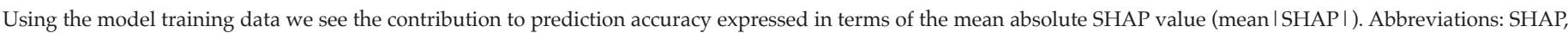

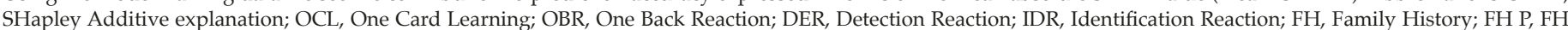

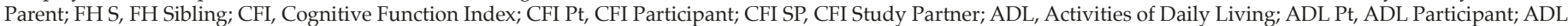
SP, ADL Study Partner; PACC, Preclinical Alzheimer Cognitive Composite

(DSST), and Free and Cued Selective Reminding Test (FCSRT) (12).

\section{Statistical Analysis}

Extreme Gradient Boosting (XGBoost) (4) is a decision tree-based machine learning technique (6). A single decisions tree, or regression tree, is easy to interpret but provides relatively poor prediction. Aggregating a large number of trees can improve prediction accuracy. Boosting is a technique in which models are trained in sequence, with each new model making cumulative improvements. At each iteration the data are re-weighted such that misclassified data points receive larger weights. XGBoost is a scalable tree boosting algorithm, that is optimized and designed to be highly efficient, flexible, and portable.

XGBoost supports monotone constraints and customized objective functions. We applied monotone constraints to predictors such as age, number of APOE 4 alleles $(0,1$ or 2$)$, and assessment scores that we expect to have a generally monotonic relationship with amyloid PET SUVR (Supplemental Figure 1). The 
Table 2. Operating characteristics of screening algorithms using the test data with $\mathrm{A} \beta+$ set to SUVR $\geq 1.15$

\begin{tabular}{|c|c|c|c|c|c|c|c|}
\hline Model & SUVR Threshold & Accuracy & Sensitivity & Specificity & NPV & PPV & NNS \\
\hline Remote & 1.05 & $54.88 \%$ & $60.70 \%$ & $52.77 \%$ & $78.72 \%$ & $31.81 \%$ & 3.14 \\
\hline Remote with APOE & 1.04 & $61.86 \%$ & $74.24 \%$ & $57.37 \%$ & $85.99 \%$ & $38.72 \%$ & 2.58 \\
\hline In Clinic with APOE & 1.04 & $62.33 \%$ & $73.80 \%$ & $58.16 \%$ & $85.95 \%$ & $39.03 \%$ & 2.56 \\
\hline In Clinic PACC & 1.04 & $56.63 \%$ & $58.95 \%$ & $55.78 \%$ & $78.92 \%$ & $32.61 \%$ & 3.07 \\
\hline Remote & 1.15 & $71.98 \%$ & $10.04 \%$ & $94.45 \%$ & $74.31 \%$ & $39.66 \%$ & 2.52 \\
\hline Remote with APOE & 1.15 & $77.09 \%$ & $35.81 \%$ & $92.08 \%$ & $79.81 \%$ & $62.12 \%$ & 1.61 \\
\hline In Clinic & 1.15 & $72.44 \%$ & $5.68 \%$ & $96.67 \%$ & $73.85 \%$ & $38.24 \%$ & 2.62 \\
\hline In Clinic with APOE & 1.15 & $76.51 \%$ & $36.24 \%$ & $91.13 \%$ & $79.75 \%$ & $59.71 \%$ & 1.67 \\
\hline In Clinic PACC & 1.15 & $73.60 \%$ & $6.55 \%$ & $97.94 \%$ & $74.28 \%$ & $53.57 \%$ & 1.87 \\
\hline
\end{tabular}

The top half of the table provides operating characteristics when a threshold is applied to predicted amyloid PET SUVR that results in a 50\% prediction prevalence (half of the screening pool is predicted positive and tested with a PET scan). The first column indicates the threshold required to attain $50 \%$ prediction prevalence. The bottom half of the table applies a threshold of 1.15, which reduces Number Need to Screen (NNS), but also greatly reduces sensitivity. The NNS is the inverse of the Positive Predictive Value (PPV). The PPV indicates the percentage of participants that are truly positive when the model indicates them as positive. Likewise, the Negative Predictive Value (NPV), this gives the probability that a participant is truly amyloid negative when the model indicates them as negative.

Table 3. Demographic characteristics of amyloid positive selections from the test data with A $\beta+$ set to SUVR $\geq 1.15$

\begin{tabular}{|c|c|c|c|c|c|c|c|c|}
\hline \multirow[b]{2}{*}{ Model } & \multirow[b]{2}{*}{ SUVR Threshold } & \multicolumn{3}{|c|}{ APOE4 } & \multicolumn{2}{|c|}{ Family History } & \multicolumn{2}{|c|}{ Sex } \\
\hline & & 0 & 1 & 2 & No & Yes & Female & Male \\
\hline Remote & 1.05 & $298(68.19 \%)$ & $123(28.15 \%)$ & $16(3.66 \%)$ & $140(32.04 \%)$ & $297(67.96 \%)$ & $248(56.75 \%)$ & $189(43.25 \%)$ \\
\hline In Clinic & 1.04 & $283(66.59 \%)$ & $128(30.12 \%)$ & $14(3.29 \%)$ & $112(26.35 \%)$ & $313(73.65 \%)$ & $248(58.35 \%)$ & $177(41.65 \%)$ \\
\hline In Clinic with APOE & 1.04 & $175(40.42 \%)$ & $228(52.66 \%)$ & $30(6.93 \%)$ & $120(27.71 \%)$ & $313(72.29 \%)$ & $240(55.43 \%)$ & $193(44.57 \%)$ \\
\hline Remote & 1.15 & $39(67.24 \%)$ & $17(29.31 \%)$ & $2(3.45 \%)$ & $6(10.34 \%)$ & $52(89.66 \%)$ & $27(46.55 \%)$ & $31(53.45 \%)$ \\
\hline Remote with APOE & 1.15 & $6(4.55 \%)$ & $96(72.73 \%)$ & $30(22.73 \%)$ & $30(22.73 \%)$ & $102(77.27 \%)$ & $67(50.76 \%)$ & $65(49.24 \%)$ \\
\hline In Clinic & 1.15 & $26(76.47 \%)$ & $8(23.53 \%)$ & $0(0.00 \%)$ & $7(20.59 \%)$ & $27(79.41 \%)$ & $15(44.12 \%)$ & $19(55.88 \%)$ \\
\hline In Clinic with APOE & 1.15 & $22(15.83 \%)$ & $88(63.31 \%)$ & $29(20.86 \%)$ & $34(24.46 \%)$ & $105(75.54 \%)$ & $67(48.2 \%)$ & $72(51.80 \%)$ \\
\hline
\end{tabular}

The top half of the table provides demographic characteristics when a threshold is applied to predicted amyloid PET SUVR that results in a 50\% prediction prevalence (half of the screening pool is predicted positive and tested with a PET scan). The first column indicates the threshold required to attain 50\% prediction prevalence. The bottom half of the table applies a threshold of 1.15. We can see in all the scenarios where APOE is included in the model, at least 29 of the 30 participants with APOE4 2 allele (in the test data) have been selected.

default XGBoost objective function is mean squared loss, meaning decision trees are selected to minimize the residual sum of squares. Because XGBoost does not provide confidence intervals with mean squared loss, we applied the Quantile Regression loss function to estimate the $50 \%, 2.5 \%$, and $97.5 \%$ quantiles of the predictions. XGBoost model has a number of hyperparameters that are used to assist in the issue known as the bias-variance trade-off (13). Hyper-parameters are fixed before the model is fitted and are not learned from data. We used 10-fold Cross-Validation (CV) to assess the out-of-sample bias and variance for given hyperparameter values, and Bayesian Optimization (14) to optimize the hyper-parameter selection. We use SHapley Additive exPlanation (SHAP) (15) values to summarize the importance of each predictor to the overall predictive accuracy of each model. More details about the model fitting procedures are provided in the supplemental material (Supplemental Table 1). Our main interest lies in the predictive accuracy of the models. In order to assess this, we split the data randomly into $80 \%$ training and $20 \%$ test. After fitting the models with the training data, we assess their predictive accuracy with the independent test data. Analyses were conducted with $\mathrm{R}$ version 3.6.2 (r-project.org) with packages xgboost (4) version 0.90.0.2 and mlrMBO (16) version 1.1.2. 


\section{Figure 2. ROC curves and AUCs}

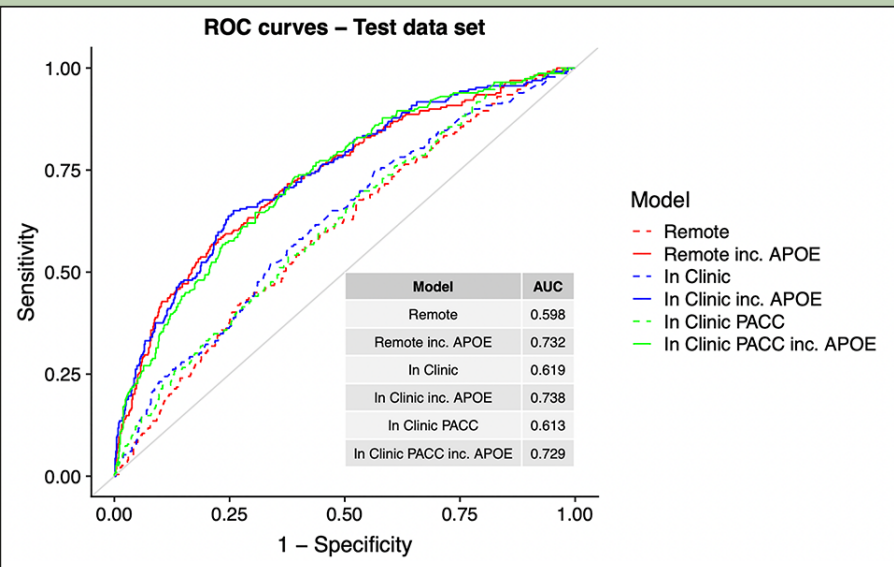

ROCs and AUCs for each model are determined using the independent test set and $\mathrm{A} \beta+$ set to SUVR $\geq 1.15$. The colors represent the setting type; Remote (red), In-Clinic (blue) and PACC components (blue). Abbreviations: ROC, Receiver Operating Characteristic; AUC, Area Under the Curve; PACC, Preclinical Alzheimer Cognitive Composite

\section{Results}

Figure 1 shows the relative contributions, in terms of SHAP values, for each predictor to the predictive accuracy of each model. As expected, when available, APOE genotype is the most important predictor for these cross-sectional models. We see that age, CFI, education, and family history also enter the top 5 most valuable predictors in some models. Figure 2, the Receiver Operating Characteristic (ROC) curves and Area under the Curve (AUC) for the 6 models, also demonstrates the relative value of APOE. The dashed lines are models fitted without the APOE 4 variable and the solid lines are for models that include APOE $\varepsilon 4$. The ROC curves were generated using a cut point SUVR value of 1.15 for a binary separation between amyloid positive and negative. In general, we see AUCs in the range 0.60 (without APOE) to 0.73 (with APOE).

Figure 3 expresses prediction accuracy in terms of screening for a clinical trial. The top panel shows 1/ Positive Predictive Value (PPV), which is equivalent to the number needed to screen (with amyloid PET) to identify one eligible participant. In this figure, movement along the horizontal axis represents varying the threshold applied to SUVRs predicted from each model. The bottom panel provides the required number of potential participants (e.g. webstudy participants) in order to identify $1,000 \mathrm{~A} \beta+$ participants.

Table 2 reports operating characteristics from several screening algorithm scenarios. The top half provides operating characteristics when a threshold is selected to provide $50 \%$ prediction prevalence (i.e. select half the participant pool to receive amyloid PET scans). With 50\% prediction prevalence, the NNS is about 2.5 participants with APOE and 3.0 participants without APOE. When the threshold for predicted amyloid PET is increased to 1.15 , the NNS is reduced to about 1.7 participants with
APOE and 2.5 participants without APOE. However, this results in much lower sensitivity, and as we can see from Figure 3, a threshold of 1.15 would be practical only with participant registries of $10,000-13,000$ to identify 1,000 $\mathrm{A} \beta+$ participants.

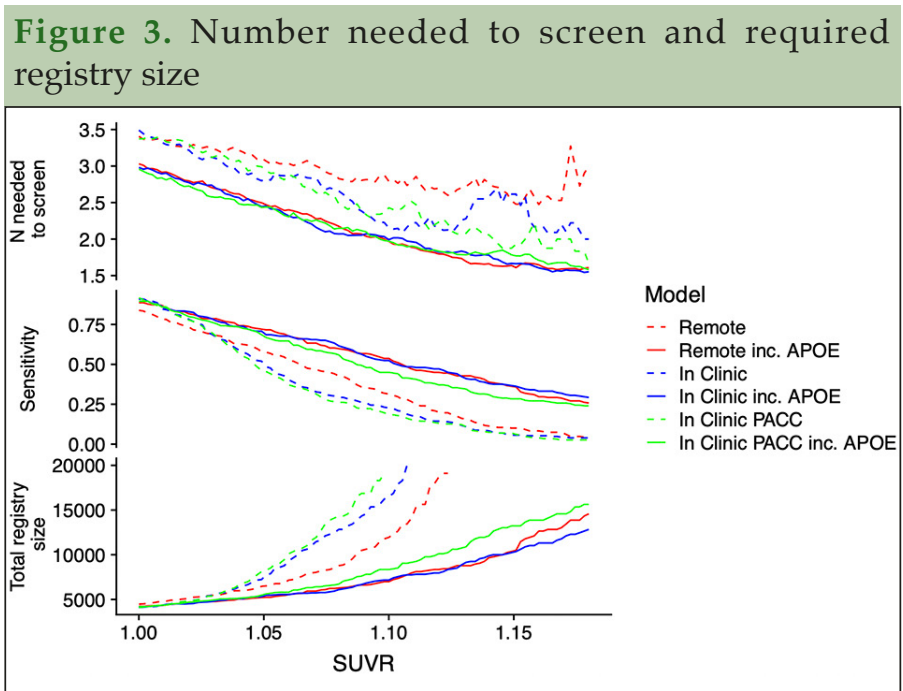

The top panel shows the number needed to screen (which is equivalent to $1 / \mathrm{PPV}$ with amyloid PET to identify one $\mathrm{A} \beta+$ individual by applying the given SUVR threshold to the values predicted from each model. The middle panel shows sensitivity. The models not containing APOE\&4 all have lower sensitivity. The bottom panel shows the size of the screening pool (e.g. web-based registry) that would be required to recruit $1,000 \mathrm{~A} \beta+$ individuals by applying the given SUVR threshold to values predicted from each model.

Abbreviations: PPV, Positive Predictive Value; SUVR, Standardized Uptake Value Ratio; PACC, Preclinical Alzheimer Cognitive Composite; PET, positron emission tomography

\section{Discussion}

This work, in the context of the TRC-PAD platform, can facilitate the development of participant selection algorithms. TRC-PAD has two main selection points; the first is from the APT webstudy to in-clinic assessment (stage 1) and the second is from in-clinic to amyloid testing (stage 2). In stage 1, consented webstudy participants are referred to their nearest TRC-PAD site, identified via the use of self-reported zip codes. They are then ranked based on their SUVR prediction. In addition to this predicted SUVR, the selection process considers demographics to achieve diversity and if the participant has known prior amyloid testing and results. During the first in-clinic visit of the referred participants in stage 1 , additional cognitive testing, in the form of the PACC, and APOE genotyping is performed. With this additional information, the SUVR predictions are updated and presented for central authorization of amyloid testing.

This work has shown that by collecting relatively simple demographics, cognitive and functional assessments remotely, via the webstudy, we will be able to reduce screen fail rates and improve enrollment. Even small improvements in NNS can have a large impact on the expense of screening for Preclinical AD clinical trials. For example, assuming a conservative estimate of 3,500 US Dollars (USD) per scan, the A4 study spent 
a total of about $4,486 \times 3,500$ (USD) $=15,701,000$ (USD) for screening amyloid PET scans alone to identify $1,323 \mathrm{~A} \beta+$ individuals (NNS=3.39). Reducing the NNS from 3.39 to 2.62 , which seems plausible with the simplest remote battery, would have reduced this cost by $3,569,090$ (USD) to $1,323 \times 2.62 \times 3,500$ (USD) $=12,131,910$ (USD). In addition to the remote data setting, this work included the value of APOE genotyping and collection of PACC during an in-clinic screening. Adding APOE genotype might reduce NNS to below 2.00, for a total PET screening cost of $1,323 \times 2.00 \times 3,500$ (USD) $=9,261,000$ (USD). The financial impact would be less with a cerebrospinal fluid (CSF)based, or blood-based, amyloid screen, but the impact on subject and site burden would remain significant. From a statistical aspect, we have demonstrated the use of Machine Learning Techniques to both optimize, via Bayesian Optimization, and produce predictive models using XGBoost. We have illustrated how to make inferences from a modelling approach that is primarily used for prediction via the SHAP metric.

One limitation of these pre-screening algorithms is that the cohort characteristics will be impacted. For example, we would expect the algorithms to produce an older cohort with an even greater proportion of APOE 4 carriers than a cohort selected without a pre-screen. This could be mitigated by stratifying the screening process to ensure an adequate sample of younger, APOE 44 noncarriers; but with adverse effects on the NNS. Another consideration is the inability for these models to extrapolate beyond the data in the continuous variables such as age. A second potential limitation is in the bias of the training data. As we start using these models in TRCPAD and collect additional data, we will assess whether the models are biased against any additional covariates collected.

Future work will focus on utilizing longitudinal cognitive and functional change and/or the use of bloodbased biomarkers to improve the performance of these predictive models and algorithms. We anticipate, based on analyses of the Alzheimer Disease Neuroimaging Initiative (ADNI) (5), that longitudinal change may be a valuable predictor of amyloid status. In addition, we will incorporate plasma amyloid peptide ratios (currently in validation testing) into the final stage of prediction and expect a large improvement in prediction.

Acknowledgements: We thank the A4 Study Team for their data sharing policy (NIA grants U19AG010483 and R01AG063689). Without the use of such a rich dataset we would not be able to conduct this research. We would also like to thank the Alzheimer's Therapeutic Research Institute (ATRI) and the members that make up the TRC-PAD project. Dr Cummings is supported by Keep Memory Alive (KMA); NIGMS grant P20GM109025; NINDS grant U01NS093334; and NIA grant R01AG053798.

Ethical standard: Institutional Review Boards (IRBs) approved these studies, and all participants gave informed consent before participating.

Conflict of interest: Dr. Raman reports grants from National Institute on Aging, grants from Eli Lilly, during the conduct of the study. Dr. Sperling reports personal fees from AC Immune, personal fees from Biogen, personal fees from Janssen, personal fees from Neurocentria, personal fees from Eisai, personal fees from GE Healthcare, personal fees from Roche, personal fees from
InSightec, personal fees from Cytox, personal fees from Prothena, personal fees from Acumen, personal fees from JOMDD, personal fees from Renew, personal fees from Takeda Pharmaceuticals, personal fees from Alnylam Pharmaceuticals, personal fees from Neuraly, grants from Eli Lilly, grants from Janssen, grants from Digital Cognition Technologies, grants from Eisai, grants from NIA, grants from Alzheimer's Association, personal fees and other from Novartis, personal fees and other from AC Immune, personal fees and other from Janssen, outside the submitted work. Dr. Cummings has provided consultation to Acadia, Actinogen AgeneBio, Alkahest, Alzheon, Annovis, Avanir, Axsome, Biogen, BioXcel Cassava, Cerecin, Cerevel, Cortexyme, Cytox, EIP Pharma, Eisai, Foresight, GemVax, Genentech, Green Valley, Grifols, Karuna, Merck, Novo Nordisk, Otsuka, Resverlogix, Roche, Samumed, Samus, Signant Health, Suven, Third Rock, and United Neuroscience pharmaceutical and assessment companies.Dr. Cummings has stock options in ADAMAS, AnnovisBio, MedAvante, BiOasis. Dr. Cummings owns the copyright of the Neuropsychiatric Inventory. Dr Cummings is supported by Keep Memory Alive (KMA); NIGMS grant P20GM109025; NINDS grant U01NS093334; and NIA grant R01AG053798. Mrs. Jimenez-Maggiora, Langford, and Sun report grants from National Institutes of Health (NIH) National Institute on Aging Grant number: R01AG053798, during the conduct of the study. Dr. Aisen reports grants from Janssen, grants from NIA, grants from FNIH, grants from Alzheimer's Association, grants from Eisai, personal fees from Merck, personal fees from Biogen, personal fees from Roche, personal fees from Lundbeck, personal fees from Proclara, personal fees from Immunobrain Checkpoint, outside the submitted work. Dr. Donohue reports grants from National Institutes of Health (NIH) National Institute on Aging Grant number: R01AG053798, during the conduct of the study; personal fees from Biogen, personal fees from Roche, personal fees from Neurotrack, personal fees from Eli Lilly, other from Janssen, outside the submitted work.

Open Access: This article is distributed under the terms of the Creative Commons Attribution 4.0 International License (http:/ / creativecommons.org/ licenses/by/4.0/), which permits use, duplication, adaptation, distribution and reproduction in any medium or format, as long as you give appropriate credit to the original author(s) and the source, provide a link to the Creative Commons license and indicate if changes were made.

\section{References}

1. Sperling RA, Donohue MC, Raman R, et al. Association of Factors With Elevated Amyloid Burden in Clinically Normal Older Individuals. JAMA Neurol. 2020.

2. Aisen PS, Sperling RA, J Cummings J, et al. The Trial-Ready Cohort for Preclinical/prodromal Alzheimer's Disease (TRC-PAD) Project: An Overview. J Prev Alz Dis 2020;4(7):208-212

3. Donohue MC, Sperling RA, Salmon DP, et al. The Preclinical Alzheimer Cognitive Composite: measuring amyloid-related decline. JAMA neurology. 2014;71(8):961-970.

4. Chen T, He T, Benesty M, et al. xgboost: Extreme Gradient Boosting. 2018.

5. Insel PS, Palmqvist S, Mackin RS, et al. Assessing risk for preclinical $\beta$-amyloid pathology with APOE, cognitive, and demographic information. Alzheimer's \& Dementia: Diagnosis, Assessment \& Disease Monitoring. 2016;4:76-84.

6. Breiman L. Random forests. Machine learning. 2001;45(1):5-32.

7. Sperling RA, Rentz DM, Johnson KA, et al. The A4 study: stopping AD before symptoms begin? Science translational medicine. 2014;6(228):228fs213.

8. Sperling RA, Donohue MC, Raman R, et al. Factors associated with elevated amyloid burden in cognitively unimpaired older individuals: Screening Amyloid PET results from the A4 Study. Submitted.

9. Maruff P, Thomas E, Cysique L, et al. Validity of the CogState brief battery: relationship to standardized tests and sensitivity to cognitive impairment in mild traumatic brain injury, schizophrenia, and AIDS dementia complex. Archives of Clinical Neuropsychology. 2009;24(2):165-178.

10. Walsh SP, Raman R, Jones KB, Aisen PS. ADCS Prevention Instrument Project: the Mail-In Cognitive Function Screening Instrument (MCFSI). Alzheimer Dis Assoc Disord. 2006;20(4 Suppl 3):S170-178.

11. Folstein MF, Folstein SE, McHugh PR. "Mini-mental state": a practical method for grading the cognitive state of patients for the clinician. Journal of psychiatric research. 1975;12(3):189-198.

12. Buschke H. Cued recall in amnesia. J Clin Neuropsychol. 1984;6(4):433-440.

13. James G, Witten D, Hastie T, Tibshirani R. An introduction to statistical learning. Vol 112: Springer; 2013.

14. Snoek J, Larochelle H, Adams RP. Practical bayesian optimization of machine learning algorithms. Paper presented at: Advances in neural information processing systems 2012.

15. Lundberg SM, Lee S-I. Consistent feature attribution for tree ensembles. arXiv preprint arXiv:170606060. 2017

16. Bischl B, Richter J, Bossek J, Horn D, Thomas J, Lang M. mlrMBO: a modular framework for model-based optimization of expensive black-box functions. arXiv preprint arXiv:170303373. 2017. 